\section{Size of stimulus set and response latency in visual search*}

\author{
GEORGE C. STONE and ROBERT A. SOLEM \\ Langley Porter Neuropsychiatric Institute, San Francisco, Calif. 94122 \\ and \\ University of California in San Francisco, Calif. 94122
}

The effect of stimulus set size was studied in a visual search task called "matching-to-sample." Ss' task was to locate a predetermined "matching" stimulus in a display of similar stimuli. It was found that increasing the number of different stimuli seen on previous trials, while holding display size constant, increased reaction time (RT) to identical displays. This result is discussed in relation to feature-testing models of stimulus processing.

Speed of stimulus recognition appears to depend on the expectations of the perceiver. For example, in choice reaction time (CRT), it has been repeatedly demonstrated that response time (RT) is a function of the size $[\mathrm{m}(\mathrm{S})]$ of the stimulus set from which stimuli are drawn from trial to trial (Hick, 1952; for recent reviews, see Welford, 1960, and Smith, 1968). However, efforts to attribute this " $\mathrm{m}(\mathrm{S})$ " effect to the stimulus-processing or response-selection portions of the CRT task have been equivocal (Smith, 1968).

The present study investigated the effect of $m(S)$ on $R T$ using a task, matching-to-sample (MTS), that avoids this analytic difficulty. On a given MTS trial, items in a display are compared with a "sample" registered in memory just prior to display onset. The task is to locate a copy of the sample in the display and press a transparent response panel located in front of the stimulus. Since the response is directed at the target, problems of s-r compatibility and response categorization are minimal in this task.

MTS is conceptualized as consisting of a set of comparisons of display items with the sample until the outcome of a comparison is a "match." Previous experiments in our laboratory have shown that RT is a linear function of display size (Stone, 1970). These findings indicate that comparisons are carried out serially until a match is found.

We entertained two opposing hypotheses about the relationship of nonpresent items to individual comparisons, when display size is held constant. If increasing the stimulus set does not increase RT, then Ss'

*This work was partially supported by DMH Grant 69-1C and GRSG Grant FR-05550 from NIH-USPHS to Langley Porter Neuropsychiatric Institute and FR-00122-2-447-620-23762 to the Computer Center of the University of California at San Francisco. This paper does not reflect the views or policies of the California Department of Mental Hygiene. information for determining a "match" or the characteristics of the particular sample and display item being compared (as, for example, in a template-matching model, Hick, 1952). On the other hand, if increasing the stimulus set does increase RT (for the same display), then it logically follows that nonpresent stimuli do influence RT. The present experiment was designed to test whether performance on identical displays would show a longer RT when embedded in a series of trials utilizing a larger stimulus set.

\section{METHOD}

Two stimulus sets were created such that the items in the smaller set $[\mathrm{m}(\mathrm{S})=6]$ were a subset of the larger set $[\mathrm{m}(S)=14]$. The sets were composed of compound forms generated from superposition of two simple geometric figures, as, for example, triangle and circle. The " $m-6$ " stimuli utilized all combinations of the following four figures: triangle, circle, vertical line, and " $X$." The " $\mathrm{m}-14$ " stimuli used all combinations of these foregoing plus the rectangle and the horizontal line, but excluding the horizontal line paired with the vertical line. On each trial, a sample and a display, which always contained four items including the "match," were randomly drawn from the appropriate set. However, stimulus program tapes for the " $m-14$ " condition were constructed so that in each block of 12 trials, the target and display items on the 3rd, 7th, and 11th trials (unknown to $S$ ) were drawn exclusively from the smaller set.

Stimuli, approximately $3 \mathrm{~cm}$ in greatest dimension, were presented on five in-line display cells mounted $7.5 \mathrm{~cm}$ apart on a console. These cells are termed S1 through $\mathrm{S} 4$ from left to right. Responses were made by pressing square, transparent panels (R1 through R5), one centered over each of the display frames, with a sixth response panel (R6) located directly beneath R3. A pressure of $8 \mathrm{oz}$ was required to close the "no-match" may be derived entirely from microswitches behind these panels. Response latencies were recorded to the nearest millisecond on paper tape.

Twelve paid Ss (eight males and four females) were recruited by bulletin-board announcements from the students and staff of a large medical center. None had previous experience with the apparatus. Each $S$ was given one training session in MTS using simple, one-element forms as stimuli.

$S$ was comfortably seated at a distance from the response panels that allowed for maximum ease of responding and that produced visual angles of about $5.10 \mathrm{deg}$ between adjacent stimuli. $S$ began a block of trials by pressing $R 1$ to turn on the first sample stimulus. When $S$ was ready, he pressed R6, whereupon the sample would switch off, followed after $200 \mathrm{msec}$ by the onset of the display of four stimuli in S2 through S5. Ss were instructed not to remove their hands from the vicinity of R6 until they had located the matching stimulus and to use the same finger (normally the index and middle as a unit) in operating both $\mathrm{R} 6$ and the target response panel. If an incorrect response panel was pressed, the display remained on until the correct response was made. After the correct response, the next sample automatically appeared in S1. A "block separator" light appeared after each block of 12 trials, allowing Ss a brief rest. An experimental session consisted of 16 blocks and lasted approximately $15 \mathrm{~min}$. Each $\mathrm{S}$ participated in eight sessions, scheduled for the same time daily. Half the Ss (Group A) received four sessions under the " $\mathrm{m}-6$ " condition followed by four sessions of " $m$-14." The order was reversed for the remaining six Ss (Group B). Thus, Session 5 represents the "transition" session for each $\mathrm{S}$.

\section{RESULTS}

Data analyzed were the median latency of the $3 \mathrm{rd}, 7 \mathrm{th}$, and 11 th trials in each block. For " $\mathrm{m} \cdot 14$ " sessions, these were the specially constructed trials using the smaller, " $\mathrm{m}-6$ " set of stimuli only. In case of an error, the trial was deleted. Thus, the analyzed data for each $S$ consisted of 16 block medians per session calculated from performance on trials on which only " $m-6$ " stimuli appeared. The block medians of Sessions 2 through 7 were then subjected to an analysis of variance using a mixed design (Myers, 1966, Chap. 8). Session 1 was eliminated from the analysis because of gross inter- and intra-S variability due to early task learning; Session 8 was eliminated in order to balance the number of " $\mathrm{m}-6$ " and "m-14" sessions for each $\mathrm{S}$ and to eliminate any possible "last session" phenomena.

Stimulus set size, sessions within $\mathrm{m}(\mathrm{S})$ 
Table 1

Mean Latencies (Msec) of Responding in Each Session by Groups $A$ and $B$

\begin{tabular}{cccccccccccc}
\hline & Condition & 1 & 2 & 3 & 4 & & Condition & 5 & 6 & 7 & 8 \\
\hline Group A & $\mathrm{m}-6$ & 899 & 744 & 740 & 687 & $\mathrm{~m}-14$ & 740 & 715 & 719 & 686 \\
Group B & $\mathrm{m}-14$ & 806 & 771 & 726 & 694 & $\mathrm{~m}-6$ & 637 & 632 & 613 & 606 \\
\hline
\end{tabular}

condition, and block number within session, formed the "within-S" or repeated measurements variables. Block number was treated as a fixed-effects variable, since a signaled midsession pause produced a significant blocks-within-session effect $\left[F=5.47, F_{.01}(15,150)=2.34\right]$

RTs in the "m-6" condition were significantly faster than in the " $\mathrm{m} \cdot 14$ " condition $[\mathrm{F}=17.75$, $\left.F_{.01}(1.10)=10.04\right]$. Ss who were exposed to " $\mathrm{m}-6$ " tapes in the first four sessions (Group A) show an average increase of $53 \mathrm{msec}$ in RT in Session 5 when switched to the " $\mathrm{m}-14$ " tapes. Conversely, for Group B Ss, the effect of switching to "m-6" tapes at Session 5 was an average 57 -msec decrease in RT.

An analysis of the sequence of block medians within Session 5 was undertaken in order to determine if the shifts to slower or faster responding could be observed within this transition session. However, the trend within this session was not different from that seen in other sessions.

The practice effects variable (session-within-condition) was significant at the .01 level $[\mathrm{F}=10.40$, $\left.F_{.01}(2,20)=5.72\right]$. This effect was the same for both groups under both conditions (Group by Session interaction, $\mathrm{F}=0.22$ : $\mathrm{m}(\mathrm{S})$ Condition by Session interaction, $F=0.56$; Group by Session by Condition interaction, $F=1.25$ ). Therefore. the effect of the difference in stimulus set size on average RT can be estimated by comparing the grand means of the " $\mathrm{m}-6$ " and " $\mathrm{m}-14$ " latencies, respectively. This estimated effect is $52 \mathrm{msec}$.

Error rate was $5.5 \%$ of all responses on which block medians were based. There was no significant difference between number of errors in " $m-6$ " and " $m-14$ " conditions (118 to 129 , respectively, out of a total of $4,6083 \mathrm{rd}$-, $7 \mathrm{th}$-, and 11 th-trial responses). A rank-order correlation between error rate of individual $S$ s and amount of upward (Group A) or downward (Group B) shift in RT between Sessions 4 and 5 was not significant (rho $=.01)$.

\section{DISCUSSION}

The interesting implication of these results appears to be the following: Stimuli encountered on previous trials but not present on a current trial influence the process of comparing display items with the sample. We favor a feature-testing model to account for these results. The idea that stimulus recognition in visual search and matching is carried out in terms literature (Gibson, Gibson, Pick, \& Osser, 1962; Neisser, 1967).

A "feature" is an attribute of a stimulus, such as "acute angle at the top," which can be considered to be in one of two states Comparison involves the testing of the value or state of each feature from a display item against the value of the corresponding feature accessed from the sample in short-term memory. If all features have the same value, the decision of the feature-testing mechanism is "match," and the approach response is selected for immediate execution. If one or more of the corresponding feature states are different, $S$ proceeds to the next item in the display. Beyond the idea that $S$ tends to test more features when faced with a larger stimulus set, this experiment provides no evidence as to the specific mechanics of a model suitable for MTS.

One attractive model is based on the hypothesis that a well-practiced $\mathrm{S}$ tends to use the same set of features from trial to trial, but requires more features the larger of features has been discussed in the (e.g., "present" or "not present").

\section{TAY WILSON, Department of Transport, Toronto, Ontario, Canada}

Sevent1'-seren second-1'ear university, students were required to choose between pairs of one-outcome gambles. The adequacy of four utility models in predicting choice behavior was tested using the $m(S)$ condition. However, under this model, we might expect the transition session of Group A to show an increase in the rate of errors early in the session followed by a gradual adjustment, reflected in increasing RT, to the new stimuli. Group B's adjustment to fewer stimuli in Session 5 might be expected to appear gradually throughout the session. Instead, adjustment appeared to be virtually immediate for both groups of Ss. This remains a problem for further study.

These results can be related to the broader concept of expectancy or psychological set. They support the notion that a human searching an environment for an object is influenced by what he might expect to see as well as by what is there.

\section{REFERENCES}

GIBSON, E. J., GIBSON, J. J., PICK, A. D., \& OSSER, H. A developmental study of the discrimination of letter-like forms. Journal of Comparative \& Physiological Psychology, 1962, 55, 897-906.

HICK. W. E. On the rate of gain of information. Quarterly Journal of Experimental Psychology, 1952, 4, 11-26.

MYERS, J. L. Fundamentals of experimental design. Boston: Allyn \& Bacon, 1966.

NEISSER, U.' Cognitive psychology. New York: Appleton-Century-Crofts, 1967.

SMITH, F. E. Choice reaction time: An analysis of the major theoretical positions. Psychological Bulletin, 1968,69, 77-110.

STONE, G. C. Response latency in human matching-to-sample. Perception \& Psychophysics, 1970, 7, 197-205.

WELFORD, A. T. The measurement of sensory-motor performance: Survey and reappraisal of twelve years' progress. Ergonomics, 1960, 3, 189-229.

\section{The effect of defensiveness and anxiety on the ability of four gambling models to predict risk-taking behavior}

the Coombs \& Bezembinder (1964) technique for several personality' subgroups. It was found that, in general. models taking into account the subjective probability of the $S$ and the subjective utility of the prize for the $S$ were more adequate as predictors of behavior than were those using objective measures of 